\title{
THE SIMULATION OF AERIAL MOVEMENT - II. A MATHEMATICAL INERTIA MODEL OF THE HUMAN BODY
}

\author{
M. R. YEADON \\ Biomechanics Laboratory, Faculty of Physical Education, University of Calgary, Canada
}

\begin{abstract}
A mathematical inertia model which permits the determination of personalized segmental inertia parameter values from anthropometric measurements is described. The human body is modelled using 40 geometric solids which are specified by 95 anthropometric measurements. A 'stadium' solid is introduced for modelling the torso segments using perimeter and width measurements. This procedure is more accurate than the use of elliptical discs of given width and depth and permits a smaller number of such solids to be used. Inertia parameter values may be obtained for body models of up to 20 segments. Errors in total body mass estimates from this and other models are discussed with reference to the unknown lung volumes.
\end{abstract}

\section{INTRODUCTION}

A computer simulation model of human airborne movement requires the input of information specifying the initial motion, the changes in body configuration during flight and the mass distribution of the human body. The initial motion and the time history of body configuration can be described using orientation angles, while the mass distribution of the body can be described using length, mass and moment of inertia descriptors of the body segments. From this input information a simulation model is able to determine the resulting motion.

The values of the orientation angles used as input may describe a hypothetical movement, or may be derived from film data of an actual performance as described in part I of this series (Yeadon, 1990a). The values of the segmental inertia parameters may be chosen to be representative of an 'average man', or may be personalized for a particular individual.

Segmental inertia parameters for an average man have been calculated using the mean values of the anthropometric measurements given by Hertzberg et al. (1954) as input to the model of Whitsett (1963). Daniels (1952) showed that if a subject is regarded as average for a particular anthropometric measurement when his measurement lies between the 35th and 65th percentiles, then not one of the 4063 subjects of Hertzberg et al. (1954) was average in all of 10 selected linear measurements. This indicates that an 'average man' may be representative of only a very small fraction of a population. As a consequence, it will be necessary to consider a range of inertia values when attempting to generalize results of computer simulations.

There are a number of experimental techniques for determining the inertia parameters of the body segments of a living individual (Bouisset and Pertuzon, 1968; Hay, 1973; Hatze, 1975; Tichonov, 1976). However, none of these techniques is suitable for determining moments of inertia of a central segment such as the pelvis, or for determining the moment of inertia of a limb about its longitudinal axis.

Mathematical models which represent the body segments using a number of geometric solids are capable of estimating values for all segmental inertia parameters. Such models have been developed by Whitsett (1963), Hanavan (1964), Jensen (1976) and Hatze (1980). Although the detail with which body segments are modelled can be quite complex, all of the above models make simplifying assumptions such as uniform density over a given cross-section. It may be expected that these assumptions will lead to systematic errors in the models. Such errors are difficult to identify since there are no criterion values of the segmental inertia parameters with which to compare the calculated values. 
The mathematical model presented in this paper is also based upon simplifying assumptions and may also be expected to contain systematic errors. Since the calculated inertia values will subsequently be used as input to a simulation model, the effect of such errors can be evaluated and there exists the possibility of adjusting the calculated values to minimize these errors. The model described below should therefore be regarded only as an initial stage in the determination of accurate segmental inertia parameter values.

\section{THE HUMAN BODY MODEL}

The inertia model described below has been designed to produce segmental values of an individual for input into the 11 segment simulation model described in parts III and IV of this series (Yeadon, 1990b; Yeadon et al., 1990). In the 11 segment simulation model it is assumed that the segments are rigid bodies and that no movement occurs at the neck, wrists or ankles. Such assumptions may be regarded as weaknesses of the model since they limit the versatility of the model. However, if it can be shown that the agreement between simulations and performances of aerial movements is good despite these simplifying assumptions, the assumptions may be regarded as adequate.

Although the inertia model is described in terms of the 11 segments of the simulation model, it should not be regarded as an 11 segment model. Each segment is composed of a number of subsegments and it is a simple matter to regard the head, hands and feet as individual segments and to output values for 16 segments. Indeed the hands and feet may each be regarded as comprising two segments so that segmental inertia parameters for 20 body segments can be calculated by the method.

\section{Geometrical representation}

Earlier models (Whitsett, 1963; Hanavan, 1964; Jensen, 1976; Hatze, 1980) have used ellipses to model cross-sections of body segments because it was mathematically convenient to do so. Figure 1 compares a cross-section of a thorax obtained by Cornelis et al. (1978) with the stadium shape of Sady et al. (1978) and an ellipse. It is apparent that the stadium bears more resemblance to the thorax cross-section than to an ellipse of equal width and depth.

Sady et al. (1978) used solids bounded by two such stadia of different dimensions to calculate volumes of body segments. Such a procedure may be expected to give a better representation than the use of cylindrical solids, since the cross-section of the stadium solid is permitted to vary. However, the formula used by Sady et al. (1978) is based upon the volume of a truncated pyramid and this gives the correct volume only when the bounding stadia have the same ratio of width to depth.

A stadium may be defined as a rectangle of width $2 t$ and depth $2 r$ with an adjoining semi-circle of radius $r$ at each end of its width (Fig. 2). The perimeter $p=4 t+2 \pi r$ and width $w=2 t+2 r$ so that $r$ and $t$ may be calculated from perimeter and width using the equations: $r=(p-2 w) /(2 \pi-4)$ and $t=(\pi w-p) /(2 \pi-4)$. It should be noted that using the perimeter and width as input measurements, rather than depth and width, reduces the error in the estimation of cross-sectional area arising from the use of a (somewhat) arbitrary geometrical shape (Yeadon, 1984).

A stadium solid bounded by parallel stadia (Fig. 3) is defined in Appendix 1 and it is shown that cross-sections which are parallel to the two bounding stadia are also stadia.

Formulae for the mass $M$, location of mass centre $\bar{z}$ and principal moments of inertia $I_{x}, I_{y}, I_{z}$ about the mass centre are derived for a stadium solid in Appendix 2. When the widths of the rectangles of the bounding stadia are zero a stadium solid becomes a truncated cone. In such a case the formulae for the inertia parameters become indeterminate. This difficulty is overcome by adding a very thin trapezium to a truncated cone so that the formulae for a stadium solid can be used.

In the inertia model all body segments, with the exception of the cranium, are represented by a number of stadium solids or truncated cones. The cranium is modelled as a semi-ellipsoid of revolution. This solid is produced by rotating a quadrant of an ellipse with semi-axes $r$ and $h$ about one axis to produce a semi-ellipsoid with base radius $r$ and height $h$. Formulae for the inertia parameters of a semi-ellipsoid are listed in standard engineering texts (e.g. Meriam, 1971). 
a

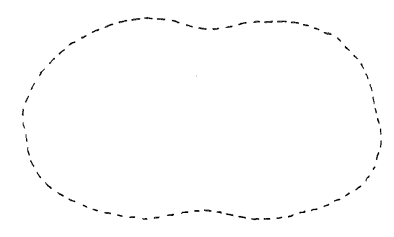

b

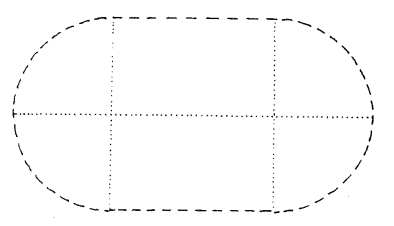

C

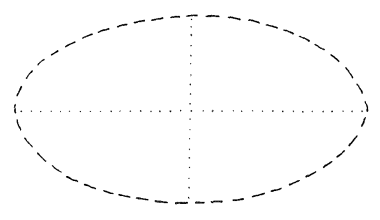

Figure 1: Comparison of (a) thorax cross-section (adapted from Cornelis et al., 1978), (b) stadium, (c) ellipse.

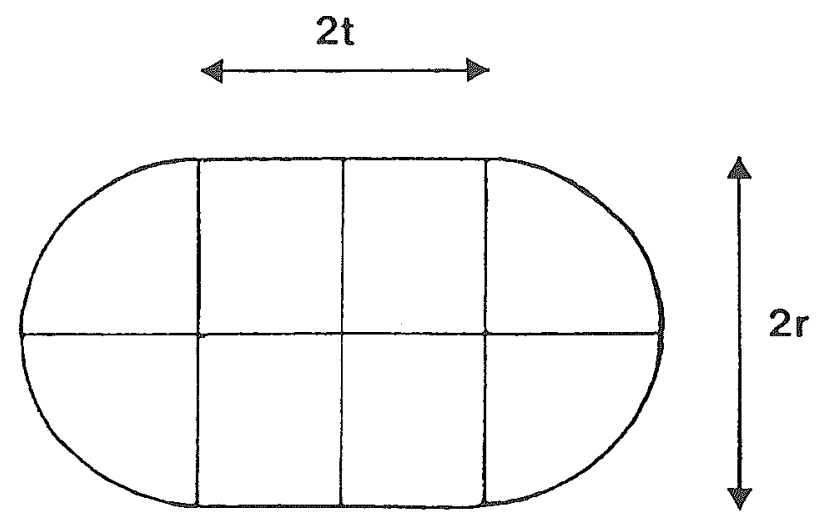

Figure 2: A stadium is a rectangle of width $2 t$ and depth $2 r$ with an adjoining semi-circle at each end of its width.

\section{Segmentation}

The body segments are sectioned into 40 solids by planes perpendicular to the longitudinal axes of the segments (Fig. 4). The levels at which the segments are sectioned are given in Table 1.

The levels $\operatorname{Lsi}(i=0,8)$ section the torso $\mathrm{S}$ into eight solids $s i(i=1,8)$ where the solid $s i$ is bounded by the levels $L s i$ and $L s(i-1)$. The left arm A and right arm B are each sectioned into seven solids $a i$ 


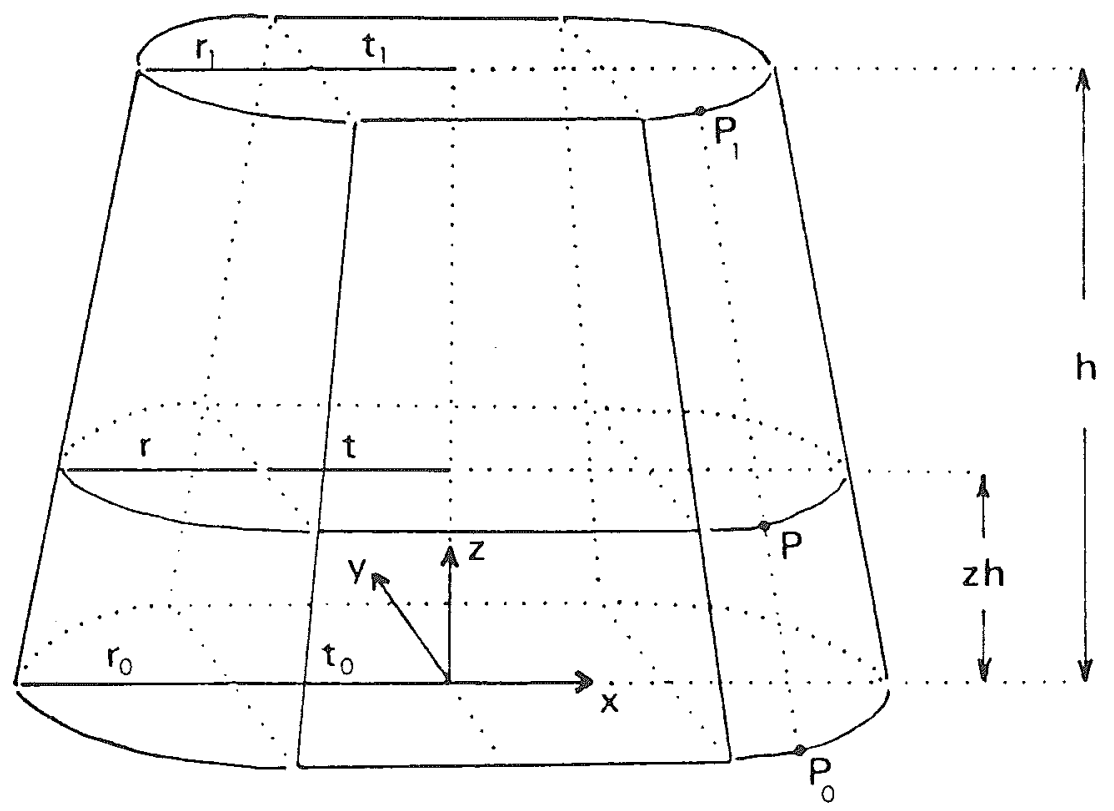

Figure 3: A stadium solid

and $b i(i=1,7)$ while the left leg $\mathrm{J}$ and right leg $\mathrm{K}$ are each sectioned into nine solids $j i$ and $k i(i=1,9)$.

The 11 segments of the simulation model of aerial movement described in part IV of this series (Yeadon et al., 1990) comprise the solids listed in Table 2.

For each segment the mass, location of mass centre, principal moments of inertia about the mass centre and distance between joint centres are calculated. It is assumed that the solids comprising a segment have coincident longitudinal axes. The values for the left and right limbs are then averaged since the simulation model is designed to have symmetrical inertia values (Yeadon et al., 1990).

\section{Measurements}

The levels in Table 1 are measured using anthropometric calipers so that the heights of the 40 solids can be calculated. At each level the perimeter is measured using a measuring tape. At the shoulder level $L s 4$ of the torso a depth measurement is taken since it is not possible to measure the perimeter directly. For the shoulder levels $L a 0, L b 0$ of the arms the perimeters are taken as high up the arms as possible.

The solids representing the head, arms and legs are assumed to be of circular cross-section so that no further measurements are needed. The remaining solids of the torso, hands and feet are stadium solids and so width measurements are taken at the boundary levels. At the heel levels $L j 6, L k 6$ anteriorposterior depths rather than medio-lateral widths are measured since these values correspond to the widths of the stadium cross-sections. The foot arch levels $L j 7, L k 7$ are assumed to be circular in crosssection to permit the transition from stadia which are aligned anterior-posteriorly to stadia which are aligned medio-laterally.

The 95 measurements taken comprise 34 lengths, 41 perimeters, 17 widths and three depths and require between 20 and 30 min of the subject's time. 
Table 1: The levels at which the body segments are sectioned

\begin{tabular}{ll}
\hline Torso & S \\
$L s 0$ & hip joint centre \\
$L s 1$ & umbilicus \\
$L s 2$ & lowest front rib \\
$L s 3$ & nipple \\
$L s 4$ & shoulder joint centre \\
$L s 5$ & acromion \\
$L s 6$ & beneath nose \\
$L s 7$ & above ear \\
$L s 8$ & top of head \\
& \\
Left & arm A \\
$L a 0$ & shoulder joint centre \\
$L a 1$ & mid-arm \\
$L a 2$ & elbow joint centre \\
$L a 3$ & maximum forearm perimeter \\
$L a 4$ & wrist joint centre \\
$L a 5$ & base of thumb \\
$L a 6$ & knuckles \\
$L a 7$ & fingernails \\
& \\
Left leg J \\
$L j 0$ & hip joint centre \\
$L j 1$ & crotch \\
$L j 2$ & mid-thigh \\
$L j 3$ & knee joint centre \\
$L j 4$ & maximum calf perimeter \\
$L j 5$ & ankle joint centre \\
$L j 6$ & heel \\
$L j 7$ & arch \\
$L j 8$ & ball \\
$L j 9$ & toe nails \\
\hline
\end{tabular}

\section{Density values}

The density values used in the inertia model are taken from Dempster (1955) and are listed in Table 3 together with the corresponding solids. These values are used in preference to those obtained in the cadaver studies of Clauser et al. (1969) and Chandler et al. (1975) since only Dempster found individual values for shoulders, thorax and pelvis. It should be noted that the value for the density of Dempster's thorax segment is used with solids $s 3$ and $s 4$ whereas segment $\mathrm{T}$ of the simulation model, which has been named the thorax segment for convenience, comprises only $s 3$.

\section{APPLICATION}

The method was applied to three subjects (two males, one female) prior to performances of twisting somersaults on trampoline. Table 4 compares total body masses, determined by weighing, with the estimates obtained using the inertia model.

\section{DISCUSSION}

The maximum error of the total body mass estimates given in Table 4 is $2.3 \%$. This value is comparable with the value $1.8 \%$ obtained by Jensen (1978) but is much greater than the value $0.5 \%$ obtained by Hatze (1980). 
Table 2:

\begin{tabular}{lcc}
\hline Segment & Symbol & Solids \\
\hline Chest-head & $\mathrm{C}$ & $s 4, s 5, s 6, s 7, s 8$ \\
Thorax & $\mathrm{T}$ & $s 3$ \\
Pelvis & $\mathrm{P}$ & $s l, s 2$ \\
Left upper arm & $\mathrm{A}_{1}$ & $a 1, a 2$ \\
Left forearm-hand & $\mathrm{A}_{2}$ & $a 3, a 4, a 5, a 6, a 7$ \\
Right upper arm & $\mathrm{B}_{1}$ & $b 1, b 2$ \\
Right forearm-hand & $\mathrm{B}_{2}$ & $b 3, b 4, b 5, b 6, b 7$ \\
Left thigh & $\mathrm{J}_{1}$ & $j 1, j 2, j 3$ \\
Left shank-foot & $\mathrm{J}_{2}$ & $j 4, j 5, j 6, j 7, j 8, j 9$ \\
Right thigh & $\mathrm{K}_{1}$ & $k 1, k 2, k 3$ \\
Right shank-foot & $\mathrm{K}_{2}$ & $k 4, k 5, k 6, k 7, k 8, k 9$ \\
\hline
\end{tabular}

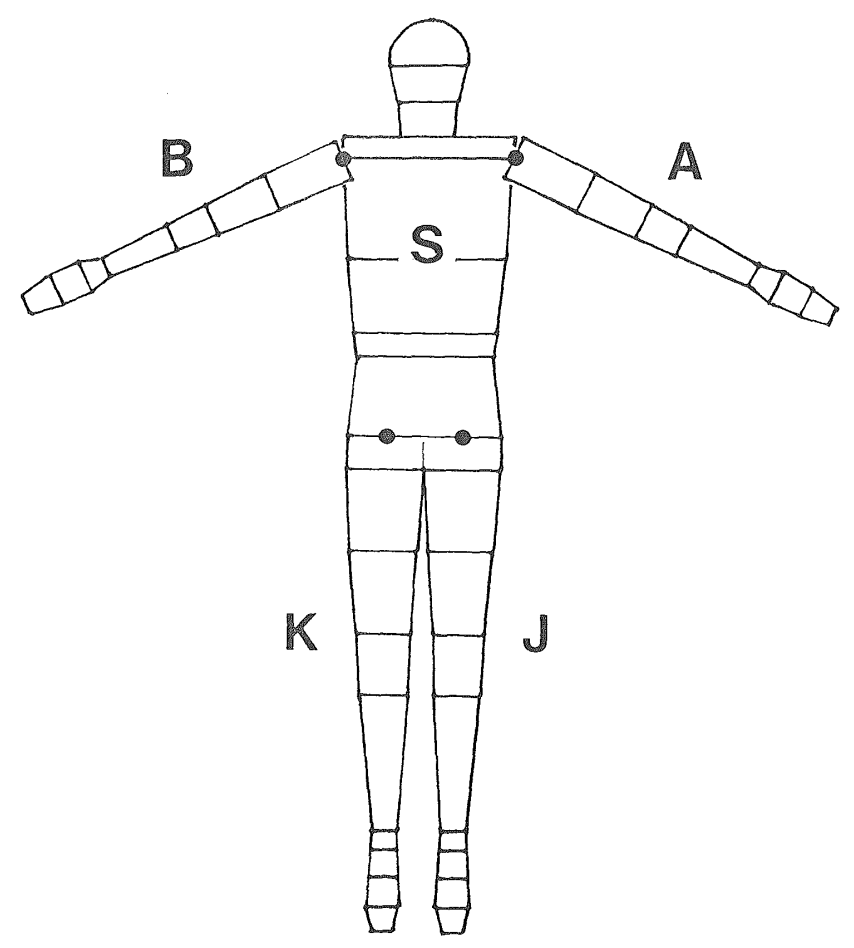

Figure 4: Sectioning of the torso S, left arm A, right arm B, left leg J and right leg K into 40 solids.

When measuring torso perimeters there is the problem of changes due to breathing. In this study an attempt was made to minimize this effect by asking the subjects to breathe shallowly. However, it may be expected that when the lungs contain an additional one litre of air, the volume of the torso increases by one litre and this will produce an increase in the total body mass estimate of about $1.5 \%$ for a 70 $\mathrm{kg}$ subject. Thus, even if the amount of air in the lungs is held approximately constant and the torso volume estimate is accurate, there appears to be an uncertainty in the torso mass estimate of the order of $1 \%$ without a method of determining the amount of air in the lungs. With this in mind, the errors in the total mass estimates of this study and of Jensen (1978) are not unreasonable.

The accuracy with which the model estimates total body mass, however, does not give much indication of the accuracy with which segmental masses and inertias are estimated. The reason for considering total body mass is that it is the only quantity for which a direct measurement is available. 
Table 3: Segmental density values (Dempster, 1955)

\begin{tabular}{lcc}
\hline \multicolumn{3}{c}{ Segmental density values $\left(\mathrm{kgl}^{-1}\right)$} \\
Segment & Solids & Density \\
\hline Head-neck & $s 6, s 7, s 8$ & 1.11 \\
Shoulders & $s 5$ & 1.04 \\
Thorax & $s 3, s 4$ & 0.92 \\
Abdomen-pelvis & $s 1, s 2$ & 1.01 \\
Upper arm & $a 1, a 2$ & 1.07 \\
Forearm & $a 3, a 4$ & 1.13 \\
Hand & $a 5, a 6, a 7$ & 1.16 \\
Thigh & $j l, j 2, j 3$ & 1.05 \\
Lower leg & $j 4, j 5$ & 1.09 \\
Foot & $j 6, j 7, j 8, j 9$ & 1.10 \\
\hline
\end{tabular}

Table 4: Accuracy of total body mass estimates

\begin{tabular}{cccc}
\hline Subject & Mass $(\mathrm{kg})$ & Estimated mass $(\mathrm{kg})$ & Error \\
\hline $\mathrm{A}$ & 60.0 & 58.8 & $-2.0 \%$ \\
$\mathrm{~B}$ & 60.9 & 62.0 & $1.8 \%$ \\
$\mathrm{C}$ & 64.3 & 65.8 & $2.3 \%$ \\
\hline
\end{tabular}

Since the inertia model has been designed to produce personalized segmental values for input into a simulation model, the inertia model may be considered adequate for this purpose providing there is good agreement between simulations and actual performances. Such evaluations will be made in parts III and IV of this series (Yeadon, 1990b; Yeadon et al., 1990) where the effects of anthropometric and film measurement errors will also be determined.

\section{References}

Bouisset, S. and Pertuzon, E. (1968). Experimental determination of the moment of inertia of limb segments. In Wartenweiler, J., Jokl, E., and Hebbelinck, M., editors, Biomechanics I, pages 107-109, Basel. Karger.

Chandler, R. F., Clauser, C. E., McConville, J. T., Reynolds, H. M., and Young, J. W. (1975). Investigation of inertial properties of the human body. Technical Report AMRL-TR-74-137, AD-AOl6-485, DOT-HS-801-430, Aerospace Medical Research Laboratories, Wright-Patterson Air Force Base, Ohio.

Clauser, C. E., McConville, J. T., and Young, J. W. (1969). Weight, volume and center of mass of segments of the human body. Technical Report AMRL-TR-69-70, Aerospace Medical Research Laboratories, Wright-Patterson Air Force Base, Ohio.

Cornelis, J., Van Gheluwe, B., Nysen, M., and Van den Berghe, F. (1978). A photographic method for the tridimensional reconstruction of the human thorax. In Coblenz, A. M. and Heron, R. E., editors, Proceedings of the Society of Photo-optical Instrumentation Engineers, volume 166, pages 294-300.

Daniels, G. S. (1952). The average man. Technical Report Technical Note WCRD-53-7, AD-10203, Wright Air Development Center, Ohio.

Dempster, W. T. (1955). Space requirements of the seated operator. Technical Report WADC-55-IS9, AD-087-892, Wright-Patterson Air Force Base, Ohio.

Hanavan, E. P. (1964). A mathematical model of the human body. Technical Report AMRL-TR-64-102, AD-608-463, Aerospace Medical Research Laboratories, Wright-Patterson Air Force Base, Ohio. 
Hatze, H. (1975). A new method for the simultaneous measurement of the moment of inertia, the damping coefficient and the location of the centre of mass of a body segment in situ. European Journal of Applied Physics, 34:217-226.

Hatze, H. (1980). A mathematical model for the computational determination of parameter values of anthropometric segments. Journal of Biomechanics, 13:833-843.

Hay, J. G. (1973). The center of gravity of the human body. In Widule, C., editor, Kinesiology III, pages 20-44, Washington, D.C. American Association or Health, Physical Education and Recreation.

Hertzberg, H. T., Daniels, E., and Churchill, E. (1954). Anthropometry of flying personnel. Technical Report WADC-TR-52-321, Wright Air Development Center, Wright-Patterson Air Force Base, Ohio.

Jensen, R. K. (1976). Model for body segment parameters. In Komi, P. V., editor, Biomechanics V-B, pages 380-386, Baltimore. University Park Press.

Jensen, R. K. (1978). Estimation of the biomechanical properties of three body types using a photogrammetric method. Journal of Biomechanics, 11:349-350.

Meriam, J. L. (1971). Dynamics, page 475. Wiley, New York.

Sady, S., Freedson, P., Katch, V. L., and Reynolds, H. M. (1978). Anthropometric model of total body volume for males of different sizes. Human Biology, 50:529-540.

Tichonov, V. N. (1976). Distribution of body masses of a sportsman. In Komi, P. V., editor, Biomechanics $V$-B, pages 103-108, Baltimore. University Park Press.

Whitsett, C. E. (1963). Some dynamic response characteristics of weightless man. Technical Report AMRL-TDR-63-18, AD-412-541, Aerospace Medical Research Laboratories, Wright-Patterson Air Force Base, Ohio.

Yeadon, M. R. (1984). The mechanics of twisting somersaults. PhD thesis, Loughborough University.

Yeadon, M. R. (1990a). The simulation of aerial movement - I. The determination of orientation angles from film data. Journal of Biomechanics, 23:59-66.

Yeadon, M. R. (1990b). The simulation of aerial movement - III. The determination of the angular momentum of the human body. Journal of Biomechanics, 23:75-83.

Yeadon, M. R., Atha, J., and Hales, F. D. (1990). The simulation of aerial movement - IV. A computer simulation model. Journal of Biomechanics, 23:85-89.

\section{Appendix 1 STADIUM SOLID}

A stadium solid bounded by parallel stadia (Fig. 3) is defined as follows: let the lower bounding stadium have parameters $r_{0}$ and $t_{0}$, the upper bounding stadium parameters $r_{1}$ and $t_{1}$ and let $h$ be the distance separating the stadia.

Consider a point $P$ which lies on the boundary of the first quadrant of a stadium with parameters $r$ and $t$ (Fig. 5). If $P$ has coordinates $(x, y)$ then:

$$
\begin{aligned}
& \text { for } \quad 0 \leq x \leq t, \quad x=\lambda t \quad(0 \leq \lambda \leq 1) \quad \text { and } y=r \\
& \text { for } \quad x>t \quad x=t+r \cos \theta \quad \text { and } \\
& \\
& y=r \sin \theta \quad(0 \leq \theta \leq \pi / 2)
\end{aligned}
$$

A correspondence may now be defined between the points on the boundaries of the first quadrants of the upper and lower stadia. Points on the straight sections correspond when they have equal $\lambda$ values and points on the curved sections correspond when they have equal $\theta$ values. In a similar way, a correspondence may be defined for the remaining quadrants. 


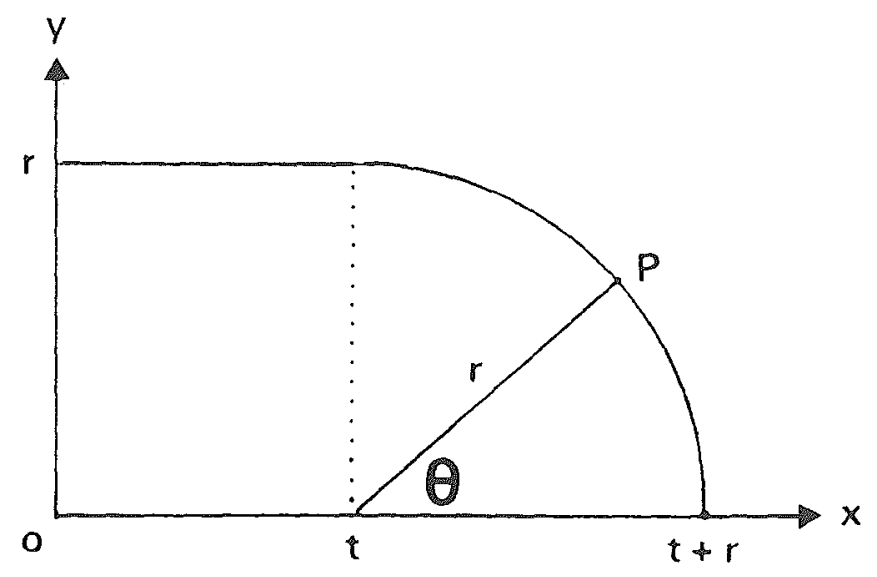

Figure 5: A quadrant of a stadium

Suppose that $P_{0}$ and $P_{1}$ are corresponding points of the lower and upper bounding stadia (Fig. 3). If $p_{0}$ and $p_{1}$ are position vectors of $P_{0}$ and $P_{1}$ relative to some origin, then the point $P$ with position vector $p=(1-z) p_{0}+z p_{1}$ lies on the line segment $P_{0} P_{1}$ when $0 \leq z \leq 1$ and divides it in the ratio $z:(1-z)$. If $z$ is held constant and $P_{0}$ and $P_{1}$ move around the lower and upper stadia, the point $P$ moves around the boundary of an intermediate section of the stadium solid. This procedure defines the stadium solid and it will now be shown that each intermediate section is itself a stadium and is parallel to the bounding stadia.

Let the coordinate system $(x, y, z)$ have origin at the centre of the lower stadium with the $z$ axis lying along the line joining the centres of the bounding stadia (Fig. 3).

The points $P_{0}\left(\lambda t_{0}, r_{0}, 0\right)$ and $P_{1}\left(\lambda t_{1}, r_{1}, h\right)$ on the straight sections correspond to $P\left((1-z) \lambda t_{0}+\right.$ $\left.z \lambda t_{1},(1-z) r_{0}+z r_{1}, z h\right)$ which is equivalent to $P(\lambda t(z), r(z), z h)$ where $t(z)=(1-z) t_{0}+z t_{1}$, and $r(z)=(1-z) r_{0}+z r_{1}$.

Similarly, the points $P_{0}\left(t_{0}+r_{0} \cos \theta, r_{0} \sin \theta, 0\right)$ and $P_{1}\left(t_{1}+r_{1} \cos \theta, r_{1} \sin \theta, h\right)$ correspond to $P(t(z)+r(z) \cos \theta, r(z) \sin \theta, z h)$ where $t(z)$ and $r(z)$ are as above.

Thus, as $P_{0}$ and $P_{1}$ move around the bounding stadia the point $P$ moves around a parallel stadium which has parameters $t(z)$ and $r(z)$ and is at a distance $z h$ from the lower bounding stadium.

It should be noted that although the above treatment does not assume that the $z$ axis is normal to the bounding stadia, only right stadium solids will be used.

\section{Appendix 2 FORMULAE FOR THE INERTIA PARAMETERS OF A STADIUM SOLID}

The derivations of formulae for the location of mass centre, mass and principal moments of inertia of a stadium solid are given below. Prior to consideration of the solid, expressions are derived for the second moments of area or a stadium.

\section{Second moments of area of a stadium}

The calculation of the second moments of area of a stadium will require the evaluation of a number of integrals. For convenience these definite integrals will be evaluated prior to the calculation of second moments. 


$$
\begin{aligned}
\int_{0}^{1 / 2 \pi} \sin ^{2} \theta d \theta & =\int_{0}^{1 / 2 \pi} \frac{1}{2}(1-\cos 2 \theta) d \theta \\
& =\frac{1}{2}\left[\theta-\frac{\sin 2 \theta}{2}\right]_{0}^{1 / 2 \pi}=\frac{1}{4} \pi \\
\int_{0}^{1 / 2 \pi} \sin ^{4} \theta d \theta & =\int_{0}^{1 / 2 \pi} \frac{1}{4}(1-\cos 2 \theta)^{2} d \theta \\
& =\frac{1}{4} \int_{0}^{1 / 2 \pi}\left(1-2 \cos 2 \theta+\cos ^{2} 2 \theta\right) d \theta \\
& =\frac{1}{4} \int_{0}^{1 / 2 \pi}\left[1-2 \cos 2 \theta+\frac{1}{2}(1+\cos 4 \theta)\right] d \theta \\
& =\frac{1}{4}\left[\frac{3 \theta}{2}\right]_{0}^{1 / 2 \pi} \\
& =\frac{3 \pi}{16} \\
\int_{0}^{1 / 2 \pi} \sin ^{2} \theta \cos \theta d \theta & =\int_{0}^{1 / 2 \pi} \sin ^{2} \theta \cdot d(\sin \theta) \\
& =\left[\frac{1}{3} \sin ^{3} \theta\right]_{0}^{1 / 2 \pi}=\frac{1}{3} \\
\int_{0}^{1 / 2 \pi} \cos ^{2} \theta \sin ^{2} \theta d \theta & =\int_{0}^{1 / 2 \pi}\left(1-\sin ^{2} \theta\right) \sin ^{2} \theta d \theta \\
& =\int_{0}^{1 / 2 \pi} \sin ^{2} \theta d \theta-\int_{0}^{1 / 2 \pi} \sin ^{4} \theta d \theta \\
& =\frac{\pi}{4}-\frac{3 \pi}{16}=\frac{\pi}{16} .
\end{aligned}
$$

A point lies on the boundary of the first quadrant of a stadium (Fig. 5) providing either $0 \leq x \leq t$ and $y=r$ or $x=t+r \cos \theta$ and $y=r \sin \theta(0 \leq \theta \leq \pi / 2)$.

Thus the area of the stadium is $A$ where:

$$
\begin{aligned}
\frac{1}{4} A & =\int_{0}^{t} y d x+\int_{1 / 2 \pi}^{0} y \dot{x} d \theta \quad \text { where } \dot{x}=\frac{d x}{d \theta} \\
& =\int_{0}^{t} r d x+r^{2} \int_{0}^{1 / 2 \pi} \sin ^{2} \theta d \theta \\
& =r t+\frac{1}{4} r^{2} \pi .
\end{aligned}
$$

Thus $A=4 r t+\pi r^{2}$ as expected.

Let $J_{x}, J_{y}$ and $J_{z}$ be the second moments of area of the stadium in Fig. 5 about the $x, y$ and $z$ axes where the $z$ axis is orthogonal to both the $x$ and $y$ axes.

$$
J_{x}=4 \int \frac{1}{3} y^{2} \cdot y d x=\frac{4}{3} \int y^{3} d x .
$$

the integration occurring over a single quadrant. Now

$$
\begin{aligned}
\int y^{3} d x & =\int_{0}^{t} y^{3} d x+\int_{1 / 2 \pi}^{0} y^{3} \dot{x} d \theta \\
& =\int_{0}^{t} r^{3} d x+r^{4} \int_{0}^{1 / 2 \pi} \sin ^{4} \theta d \theta \\
& =r^{3} t+3 \pi r^{4} / 16
\end{aligned}
$$


Thus

$$
\begin{aligned}
& J_{x}=4 r^{3} t / 3+\pi r^{4} / 4, \\
& J_{y}=4 \int x^{2} y d x
\end{aligned}
$$

and

$$
\begin{aligned}
\int x^{2} y d x & =\int_{0}^{t} x^{2} y d x+\int_{1 / 2 \pi}^{0} x^{2} y \dot{x} d \theta \\
& =\int_{0}^{t} x^{2} r d x+\int_{0}^{1 / 2 \pi}(t+r \cos \theta)^{2} \cdot r^{2} \sin ^{2} \theta d \theta \\
& =r\left[x^{3} / 3\right]_{0}^{t}+r^{2} t^{2} \int_{0}^{1 / 2 \pi} \sin ^{2} \theta d \theta+2 r^{3} t \int_{0}^{1 / 2 \pi} \sin ^{2} \theta \cos \theta d \theta+r^{4} \int_{0}^{1 / 2 \pi} \cos ^{2} \theta \sin ^{2} \theta d \theta \\
& =r\left[t^{3} / 3\right]+r^{2} t^{2}[\pi / 4]+2 r^{3} t[1 / 3]+r^{4}[\pi / 16] .
\end{aligned}
$$

Thus

$$
J_{y}=4 r t^{3} / 3+\pi r^{2} t^{2}+8 r^{3} t / 3+\pi r^{4} / 4 .
$$

For a lamina the theorem of perpendicular axes states that

$$
J_{z}=J_{x}+J_{y}
$$

Thus

$$
J_{z}=4 r t^{3} / 3+\pi r^{2} t^{2}+4 r^{3} t+\pi r^{4} / 2 .
$$

These expressions for $A, J_{x}, J_{y}, J_{z}$ will be used in the derivations of the inertia parameter of a stadium solid.

\section{The mass and location of mass centre of a stadium solid}

It has been shown that the intermediate stadium of Fig. 3 is at a distance $z h$ from the lower bounding stadium and has parameters $r$ and $t$ where $r=r_{0}+z\left(r_{1}-r_{0}\right)$ and $t=t_{0}+z\left(t_{1}-t_{0}\right)$.

Thus $r$ and $t$ may be expressed as $r=r_{0}(1+a z)$ and $t=t_{0}(1+b z)$ where $a=\left(r_{1}-r_{0}\right) / r_{0}$ and $b=\left(t_{1}-t_{0}\right) / t_{0}$. If the intermediate stadium has density $D$, area $A(z)$ and thickness $h \delta z$ the mass will be $D A(z) h \delta z$ so that the mass of a stadium solid of uniform density is given by

$$
M=\int_{0}^{1} D A(z) h d z
$$

The first moment of mass about the lower plane is $\int_{0}^{1} h z D A(z) h d z$ and the distance $\bar{z}$ of the mass centre from the lower plane is given by:

$$
\bar{z}=\int_{0}^{1} D h^{2} z A(z) d z / M .
$$

Defining the functions $F 1, F 2, F 3$ by the equations:

$$
\begin{aligned}
F 1(a, b) & =1+(a+b) / 2+a b / 3 \\
F 2(a, b) & =\frac{1}{2}+(a+b) / 3+a b / 4 \\
F 3(a, b) & =\frac{1}{3}+(a+b) / 4+a b / 5 . \\
\text { Area } A(z) & =4 r t+\pi r^{2} \\
& =4 r_{0}(1+a z) t_{0}(1+b z)+\pi r_{0}^{2}(1+a z)^{2} \\
& =4 r_{0} t_{0}\left(1+(a+b) z+a b z^{2}\right)+\pi r_{0}^{2}\left(1+2 a z+a^{2} z^{2}\right)
\end{aligned}
$$


so that

$$
\begin{aligned}
\int_{0}^{1} A d z & =4 r_{0} t_{0}(1+(a+b) / 2+a b / 3)+\pi r_{0}^{2}\left(1+2 a / 2+a^{2} / 3\right) \\
& =4 r_{0} t_{0} F 1(a, b)+\pi r_{0}^{2} F 1(a, a) \\
M & =D h \int_{0}^{1} A d z .
\end{aligned}
$$

Thus

$$
M=D h r_{0}\left[4 t_{0} F 1(a, b)+\pi r_{0} F 1(a, a)\right]
$$

Now

$$
z A=4 r_{0} t_{0}\left(z+(a+b) z^{2}+a b z^{3}\right)+\pi r_{0}^{2}\left(z+2 a z^{2}+a^{2} z^{3}\right),
$$

so that

$$
\begin{aligned}
\int_{0}^{1} z A d z & =4 r_{0} t_{0}\left(\frac{1}{2}+(a+b) / 3+a b / 4\right)+\pi r_{0}^{2}\left(\frac{1}{2}+2 a / 3+a^{2} / 4\right) \\
& =4 r_{0} t_{0} F 2(a, b)+\pi r_{0}^{2} F 2(a, a) \\
\bar{z} & =D h^{2} \int_{0}^{1} z A d Z / M
\end{aligned}
$$

Thus

$$
\bar{z}=D h^{2}\left[4 r_{0} t_{0} F 2(a, b)+\pi r_{0}^{2} F 2(a, a)\right] / M .
$$

Note also

$$
z^{2} A=4 r_{0} t_{0}\left(z^{2}+(a+b) z^{3}+a b z^{4}\right)+\pi r_{0}^{2}\left(z^{2}+2 a z^{3}+a^{2} z^{4}\right)
$$

so that

$$
\int_{0}^{1} z^{2} A d z=4 r_{0} t_{0}\left(\frac{1}{3}+(a+b) / 4+a b / 5\right)+\pi r_{0}^{2}\left(\frac{1}{3}+2 a / 4+a^{2} / 5\right)
$$

i.e.

$$
\int_{0}^{1} z^{2} A d z=4 r_{0} t_{0} F 3(a, b)+\pi r_{0}^{2} F 3(a, a) .
$$

This result will be used in conjunction with the theorem of parallel axes in the next section to calculate moments of inertia.

\section{Moments of inertia of a stadium solid}

In this section expressions are derived for the moments of inertia $I_{x}, I_{y}, I_{z}$ of a stadium solid about principle axes through the mass centre.

The moment of inertia of a stadium solid about the $z$ axis (Fig 3) is $I_{z}=\int_{0}^{1} D J_{z} h d z$ where the second moment of areas of a stadium has been obtained as

$$
J_{z}=4 r t^{3} / 3+\pi r^{2} t^{2}+4 r^{3} t+\pi r^{4} / 2 .
$$

Thus

$$
\begin{aligned}
I_{z}= & \operatorname{Dh}\left[(4 / 3) \int_{0}^{1} r t^{3} d z+\pi \int_{0}^{1} r^{2} t^{2} d z\right. \\
& \left.+4 \int_{0}^{1} r^{3} t d z+\frac{1}{2} \pi \int_{0}^{1} r^{4} d z\right] .
\end{aligned}
$$


Define

$$
F 4(a, b)=1+(a+3 b) / 2+\left(3 a b+3 b^{2}\right) / 3+\left(3 a b^{2}+b^{3}\right) / 4+a b^{3} / 5
$$

and

$$
F 5(a, b)=1+(2 a+2 b) / 2+\left(a^{2}+4 a b+b^{2}\right) / 3+2 a b(a+b) / 4+a^{2} b^{2} / 5 .
$$

Now

$$
\begin{aligned}
r t^{3} & =r_{0} t_{0}^{3}(1+a z)(1+b z)^{3} \\
& =r_{0} t_{0}^{3}(1+a z)\left(1+3 b z+3 b^{2} z^{2}+b^{3} z^{3}\right) \\
& =r_{0} t_{0}^{3}\left(1+(a+3 b) z+\left(3 a b+3 b^{2}\right) z^{2}+\left(3 a b^{2}+b^{3}\right) z^{3}+a b^{3} z^{4}\right)
\end{aligned}
$$

so that

$$
\int_{0}^{1} r t^{3} d z=r_{0} t_{0}^{3} F 4(a, b) .
$$

Similarly

$$
\int_{0}^{1} r^{3} t d z=r_{0}^{3} t_{0} F 4(b, a),
$$

whilst

$$
\begin{aligned}
r^{2} t^{2} & =r_{0}^{2} t_{0}^{2}(1+a z)^{2}(1+b z)^{2} \\
& =r_{0}^{2} t_{0}^{2}\left(1+2 a z+a^{2} z^{2}\right)\left(1+2 b z+b^{2} z^{2}\right) \\
& =r_{0}^{2} t_{0}^{2}\left(1+(2 a+2 b) z+\left(a^{2}+4 a b+b^{2}\right) z^{2}+2 a b(a+b) z^{3}+a^{2} b^{2} z^{4}\right)
\end{aligned}
$$

so that

$$
\int_{0}^{1} r^{2} t^{2} d z=r_{0}^{2} t_{0}^{2} F 5(a, b)
$$

and

$$
\begin{aligned}
r^{4} & =r_{0}^{4}(1+a z)^{4} \\
& =r_{0}^{4}\left(1+4 a z+6 a^{2} z^{2}+4 a^{3} z^{3}+a^{4} z^{4}\right)
\end{aligned}
$$

so that

$$
\int_{0}^{1} r^{4} d z=r_{0}^{4} F 4(a, a)
$$

Thus

$$
\begin{aligned}
I_{z}= & D h\left[4 r_{0} t_{0}^{3} F 4(a, b) / 3+\pi r_{0}^{2} t_{0}^{2} F 5(a, b)\right. \\
& \left.+4 r_{0}^{3} t_{0} F 4(b, a)+\pi r_{0}^{4} F 4(a, a) / 2\right] .
\end{aligned}
$$

The moment of inertia of a stadium section about the $y$ axis (Fig. 3) is given by the theorem of parallel axes as $J_{y} D h \delta z+(h z)^{2} A D h \delta z$ where $J_{y}$ is the second moment of area, $D$ is the density, $h \delta z$ the thickness, $h z$ the distance from the $y$ axis and $A$ the area.

If $I_{y}^{0}$ denotes the moment of inertia of the stadium solid about the $y$ axis (which lies in the lower face) then:

$$
I_{y}^{0}=\int_{0}^{1} J_{y} D h d z+\int_{0}^{1}(h z)^{2} A D h d z,
$$

i.e.

$$
I_{y}^{0}=D h \int_{0}^{1} J_{y} d z+D h^{3} \int_{0}^{1} z^{2} A d z
$$

where:

$$
\begin{aligned}
\int_{0}^{1} J_{y} d z & =\int_{0}^{1}\left(4 r t^{3} / 3+\pi r^{2} t^{2}+8 r^{3} t / 3+\pi r^{4} / 4\right) d z \\
& =4 r_{0} t_{0}^{3} F 4(a, b) / 3+\pi r_{0}^{2} t_{0}^{2} F 5(a, b)+8 r_{0}^{3} t_{0} F 4(b, a) / 3+\pi r_{0}^{4} F 4(a, a) / 4
\end{aligned}
$$


using the results obtained during the calculations of $I_{z}$, and

$$
\int_{0}^{1} z^{2} A d z=4 r_{0} t_{0} F 3(a, b)+\pi r_{0}^{2} F 3(a, a)
$$

having been derived in the section on the calculation of $\bar{z}$.

If $I_{y}$ denotes the moment of inertia about a parallel axis through the mass centre then the theorem of parallel axes gives:

$$
I_{y}=I_{y}^{0}-M \bar{z}^{2} .
$$

Similarly, the moment of inertia $I_{x}^{0}$ about the $x$ axis is given by:

$$
I_{x}^{0}=\int_{0}^{1} J_{x} D h d z+\int_{0}^{1}(h z)^{2} A D h d z,
$$

ie

$$
I_{x}^{0}=D h \int_{0}^{1} J_{x} d z+D h^{3} \int_{0}^{1} z^{2} A d z
$$

where

$$
\begin{aligned}
\int_{0}^{1} J_{x} d z & =\int_{0}^{1}\left(4 r t^{3} / 3+\pi r^{4} / 4\right) d z \\
& =4 r_{0} t_{0}^{3} F 4(a, b) / 3+\pi r_{0}^{4} F 4(a, a) / 4
\end{aligned}
$$

and the moment of inertia $I_{x}$ about a parallel axis through the mass centre is given by $I_{x}=I_{x}^{0}-M \bar{z}^{2}$. 